\title{
PREVALÊNCIA DE ESPÉCIES DE Candida spp. ISOLADAS DA CAVIDADE ORAL E PRÓTESES DENTÁRIAS REMOVÍVEIS DE PACIENTES ATENDIDOS EM CLÍNICA ODONTOLÓGICA
}

Raquel Soares da Silva, Rosana Soares da Silva, Caroline Lucio Moreira, Juliana Gomes Marques, Maikiane Aparecida Nascimento, Amanda Cristina Gomes Baruta, Keren Mayara Tambalo Brasileiro, Fabiana Gouveia Straioto, Sueli Cristina Schadeck Zago, Marcus Vinicius Pimenta Rodrigues, Daniela Vanessa Moris

Universidade do Oeste Paulista - UNOESTE, Presidente Prudente, SP. e-mail: raquel.soares02@outlook.com

\section{RESUMO}

O objetivo do presente trabalho foi determinar a prevalência de colonização e infecção por Candida spp. de mucosas e próteses de pacientes atendidos na clínica odontológica de uma universidade. Foram utilizados métodos morfológicos e bioquímicos para a identificação das espécies de Candida spp, tais como, CHROMagar Candida, o microcultivo em Cornmeal Agar e o auxonograma. A prevalência de colonização por Candida spp. em usuários de próteses foi de 70,6\%. Foram isoladas 101 amostras de Candida de 68 pacientes, das quais 69,31\% foram identificadas como C. albicans e 30,69\% como Candida não-albicans. A avaliação da prevalência de colonização da cavidade oral foi realizada em função do método de higienização da prótese e apresentou menor prevalência em pacientes que utilizavam a associação da escovação e molho no hipoclorito. A prevalência de $C$. albicans foi maior que Candida não-albicans, e a higienização adequada da prótese reduziu a prevalência de colonização da cavidade oral.

Palavras chave: prótese removível, Candida, estomatite protética, Candida albicans, prevalência.

\section{PREVALENCE OF CANDIDA SPP. ISOLATED FROM THE ORAL CAVITY AND REMOVABLE DENTAL PROSTHESIS OF PATIENTS ATTENDED AT A DENTAL CLINIC}

\begin{abstract}
This study aimed to determine the prevalence of colonization and infection by Candida spp. in mucosal and prosthetics of patients attended at an odontology clinic in university. Morphological and biochemical methods were used to identify Candida spp species, such as, CHROMagar Candida, Cornmeal Agar microculture, and auxonogram. The prevalence of colonization by Candida spp. in prosthetics users was 70.6\%. Candida's samples were isolated from 68 patients, which $69.31 \%$ were identified as C. albicans and $30.69 \%$ as Candida non-albicans. The oral's cavity evaluation of colonization prevalence was made according to the method of prosthetics hygene and showed less prevalence in patients that used brushing and soak in hypochlorite in association. The prevalence of Candida albicans was higher than Candida non-albicans in prosthestics's users and the proper hygene of the prosthetics reduced the prevalence of colonization.
\end{abstract}

Keywords: removable prosthesis, Candida, denture stomatitis, Candida albicans, prevalence.

\section{INTRODUÇÃO}

Aproximadamente metade da população adulta saudável abriga as espécies de Candida spp. na cavidade bucal, sendo que condições predisponentes como deficiências imunes $^{1}$, uso de próteses totais ${ }^{2}$, desordens endócrinas, lesões de tecidos moles, medicamentos como antibióticos e corticosteroides $^{3,4}$, tabagismo ${ }^{5}$ e infecção pelo $H_{I V}{ }^{4,6,7}$ podem facilitar a invasão tissular dessa levedura ${ }^{5}$.

A estomatite protética, presente em 
aproximadamente $75 \%$ dos usuários de prótese total ${ }^{8}$, é uma manifestação crônica atrófica causada por diversos fatores como higiene oral inadequada ou ao trauma mecânico causado por condições inadequadas da prótese à estrutura dentária e pela infecção por Candida spp. (candidíase oral) ${ }^{9}$. O material utilizado na fabricação das próteses dentárias (resina e/ou materiais metálicos) são superfícies que favorecem a formação de biofilmes que é considerada uma adaptação de microorganismos para sua melhor viabilidade e multiplicação no ambiente bucal, além de importante fator de virulência dessas leveduras ${ }^{10}$.

Candida albicans é a espécie isolada com maior frequência infectando diferentes sítios anatômicos. Apresenta como principais fatores de patogenicidade e virulência a capacidade de aderência a diferentes mucosas e epitélios; o dimorfismo, com produção de pseudo-hifas que auxiliam na invasão tissular; a termotolerância e a produção de exoenzimas, como proteinases e fosfolipases. Apesar de predominância de $C$. albicans, a presença de outras espécies não-albicans em isolados da superfície de próteses dentárias e mucosa oral é um importante fator para a saúde do indivíduo ${ }^{11}$, principalmente na população idosa $^{12,13}$. Levando em consideração as informações já descritas, o presente trabalho teve como objetivo determinar a prevalência de colonização e/ou infecção por Candida spp., assim como definir a prevalência de diferentes espécies de Candida colonizadoras das próteses e mucosas de pacientes atendidos em uma clínica de odontologia de uma universidade.

\section{METODOLOGIA}

Trata-se de um estudo prospectivo de prevalência-período, no qual foram estudados 68 pacientes usuários de próteses dentárias removíveis, atendidos em uma clínica de odontologia de uma universidade. Esse estudo foi aprovado pelo Comitê de Ética e Pesquisa da Universidade do Oeste Paulista sob protocolo Plataforma Brasil: 20741113.7.0000.5515.

Os pacientes que concordaram em participar do estudo e entenderam os objetivos da pesquisa assinaram $o$ termo de consentimento livre esclarecido, responderam a um questionário e foram submetidos à coleta do material da cavidade oral e da prótese removível. Utilizaram-se critérios de inclusão e exclusão, sendo que a inclusão envolvia pacientes maiores de 18 anos e que fizessem uso de próteses removíveis e os de exclusão foram: indivíduos com HIV, uso de antifúngicos no período da coleta e menores de 18 anos.

O material da cavidade oral e da prótese foram colhidos com swabs estéreis umedecidos em solução salina e semeados em CHROMagar $^{\mathrm{TM}}$ Candida, a reação colorimétrica do meio CHROMagar permitiu a identificação presuntiva de Candida albicans e confirmação de colônias puras. A identificação foi feita por avaliação da micromorfologia em Cornmeal agar acrescido de $1,0 \%$ de tween 80 e Auxonograma, assimilação de carboidratos convencionais.

Os dados foram apresentados em tabelas de frequências absolutas (n) e percentuais (\%). As associações entre variáveis foram analisadas por meio do teste do quiquadrado, teste $G$ e teste exato de Fisher. As análises foram realizadas no programa BioEstat 5.3 utilizando nível de significância de 5\% $(p<0,05)$.

\section{RESULTADOS}

Foram estudados 68 pacientes em reabilitação atendidos em clínica de odontologia, cujas idades variavam de 44 a 89 anos, com média igual a 64,87 anos. Destes, 26 pacientes eram do sexo masculino e 42 do sexo feminino, a razão de feminilidade 1, 62:1,00 dos quais foram coletadas amostras da mucosa oral e da prótese, portanto para cada um dos pacientes foram coletadas duas amostras, totalizando 136 amostras.

Dos 68 pacientes, $76,47 \% \quad(n=52)$ apresentaram-se positivo para presença de leveduras, em mucosa oral e/ou prótese. Dentre estes, $92,31 \%(n=48)$ eram colonizados e/ou infectados por espécies de Candida, enquanto em $7,69 \% \quad(n=4)$ das amostras positivas houve presença de leveduras dos gêneros Rhodotorula e Trichosporon. A análise da prevalência de Candida spp. em todos os pacientes usuários de próteses removíveis evidenciou que $70,6 \%$ desses pacientes apresentam colonização por Candida spp.

A análise da prevalência de colonização por Candida spp, em função do método de 
higienização da prótese removível, apresentou menor prevalência em pacientes que utilizavam a associação da escovação e molho no hipoclorito. Os fatores socioeconômicos e fatores socioambientais como sexo, idade, cor, tabagismo, etilismo, diabetes, uso de antisséptico bucal, uso anterior de antifúngicos e antibióticos não revelaram associação com a colonização e/ou infecção da cavidade oral de usuários de prótese removível.

A análise da prevalência de $C$. albicans e $C$. nãoalbicans foi realizada em amostras isoladas das próteses; mucosas e próteses e mucosas e revelou menor colonização em mucosa por $C$. albicans (Tabela 1).

Tabela 1. Avaliação da colonização das amostras isoladas de próteses, mucosas e próteses e mucosas de 68 pacientes usuários de próteses removíveis.

\section{$\begin{array}{lll}\text { C. albicans C. não-albicans } & \begin{array}{c}\text { C. albicans + } \\ \text { C. não-albicans }\end{array} \text { Não Candida }\end{array}$}

\begin{tabular}{lccccccccc}
\hline Amostras & $\mathbf{n}$ & $\mathbf{\%}$ & $\mathbf{n}$ & $\mathbf{\%}$ & $\mathbf{n}$ & $\mathbf{\%}$ & $\mathbf{n}$ & $\mathbf{\%}$ & $\mathbf{p}$ \\
\hline Prótese & 11 & $68,75 \mathrm{a}$ & 0 & 0 & 3 & 18,75 & 2 & 12,5 & \\
Mucosa & 1 & $25,0 \mathrm{~b}$ & 2 & 50,0 & 0 & 0 & 1 & 25,0 & 0,0043 \\
Prótese + Mucosa & 17 & $53,12 \mathrm{a}$ & 0 & 0 & 14 & 43,75 & 1 & 3,13 & \\
\hline
\end{tabular}

Teste $\mathrm{G} p<0,05$. Letras diferentes na coluna indicam diferenças significativas.

Entre os pacientes colonizados por Candida spp., $33(68,75 \%)$ eram colonizados por apenas uma espécie, 11 (22,93\%) por duas espécies e quatro $(8,32 \%)$ por três espécies. As associações de espécies ocorreram conforme mostrado na Tabela 2.

Tabela 2. Distribuição de prevalência dos isolados em relação às associações de uma ou mais espécies em prótese e mucosas.

\begin{tabular}{lcc}
\hline \multicolumn{1}{c}{ Espécie } & \multicolumn{2}{c}{ Prevalência } \\
\hline C. albicans & N & $\%$ \\
C. parapsilosis & 29 & 60,42 \\
Candida spp & 3 & 6,25 \\
C. albicans + C. glabrata & 1 & 2,08 \\
C. albicans + C. tropicalis & 7 & 14,58 \\
C. albicans + C. parapsilosis & 1 & 2,08 \\
C. albicans + Candida spp & 1 & 2,08 \\
C. tropicalis + Candida spp & 1 & 2,08 \\
C. albicans + C. glabrata + C. tropicalis & 1 & 2,08 \\
C. albicans + C. glabrata + C. parapsilosis & 2 & 2,08 \\
C. albicans + C. tropicalis + Candida spp & 1 & 4,16 \\
Total & 48 & 100 \\
\hline
\end{tabular}

A prevalência de cada espécie de Candida foi analisada de acordo com as 101 colônias isoladas de próteses e mucosas, onde 69,31\% ( $n=70$ ) das colônias eram de C. albicans,
$13,86 \%(\mathrm{n}=14)$ de $C$. glabrata, $6,93 \%(\mathrm{n}=7)$ de $C$. parapsilosis, $5,94 \%(\mathrm{n}=6)$ de $C$. tropicalis e $3,97 \%(n=4)$ de Candida spp. (Figura 1). 


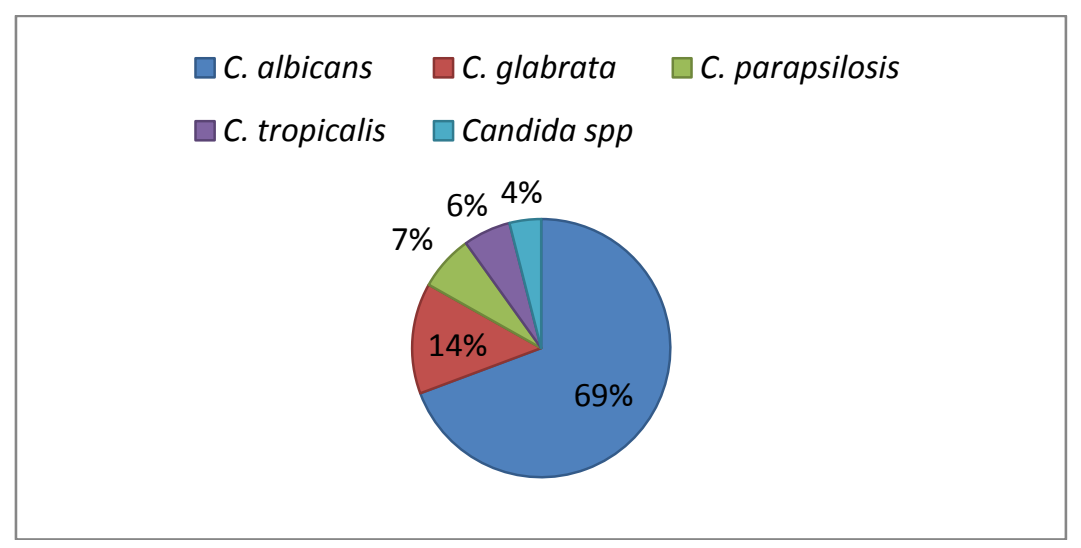

Figura 1. Distribuição de 106 isolados de próteses e mucosas obtidos de 68 pacientes usuários de próteses removíveis segundo espécie. Apresentação segundo prevalência, em porcentagem.

\section{DISCUSSÃO}

A Candida spp. é considerada uma levedura comensal da cavidade oral, a sua transformação em patógeno oportunista depende da combinação de fatores que estão relacionados ao hospedeiro, a sua atividade fúngica e alguns fatores que modificam a cavidade oral ${ }^{14,15,17}$.

A estomatite protética que acomete cerca de $65,0 \%$ dos usuários de prótese total, é caracterizada por uma lesão observada sob a área chapavel da prótese ${ }^{17,18}$, associada à alergia ao monômero residual, placa microbiana, trauma, uso continuo da prótese, hipossalivação e principalmente pela infecção por Candida spp. ${ }^{17}$, especialmente em pacientes idosos, que tendem a ser edentados, e consequentemente, possuem a microbiota modificada decorrente da ausência dos dentes ${ }^{19,20}$. A infecção por Candida spp. relaciona-se também à má adaptação, hiposalivação, desgaste pelo uso ou pela má higienização da prótese, circunstâncias essas que estão, na maioria das vezes, associadas aos indivíduos idosos ${ }^{19,20}$. Os resultados obtidos no presente estudo indicam que o uso de próteses dentárias removíveis e a higienização deficiente das próteses entre pacientes idosos atendidos na clínica de odontologia predispõem a colonização e/ou infecção por Candida spp, em concordância com os trabalhos revisados ${ }^{18,20,21}$.

Neste trabalho, a idade dos pacientes variou de 44 a 89 anos, com mediana igual a 64,00 , e média de 66,50, assim como os pacientes avaliados por Bianchi et al. ${ }^{20}$, onde a idade média do paciente foi de 72,7 anos.
No presente estudo, pode-se observar a prevalência de fungos do gênero Candida, em concordância com Teixeira e Mezzari ${ }^{22}$. A C. albicans foi a espécie predominante na colonização tanto em amostras da cavidade oral (mucosas) quanto nas próteses, confirmando publicações prévias ${ }^{20-25}$. A frequência de $C$. albicans nos pacientes colonizados variou de 76,32 a $85,02 \%$ nos trabalhos publicados ${ }^{20-25}$ e foi igual a $69,0 \%$ no presente estudo. Essas frequências não diferem entre si. Deve-se registrar que o método utilizado para identificação de $C$. albicans não permitiu sua diferenciação da $C$. dubliniensis o que poderá alterar a frequência observada no presente estudo.

A prevalência de Candida não-albicans observada no presente estudo não diferiu das relatadas por outros autores ${ }^{20-25}$. As espécies mais frequentes isoladas foram $C$. glabrata seguida da C. parapsilosis, C. tropicalis e Candida spp, além de outras leveduras de gênero não Candida. Estes resultados corroboram com estudos de Senna ${ }^{21}$. Por outro lado, Leite et al. ${ }^{23}$ e Gaspareto et al. ${ }^{24}$ referiram o isolamento de $C$. tropicalis como a segunda espécie mais frequente.

Sinais clínicos de candidíase foram observados em dez pacientes (15,62\%); e de apenas um paciente $(10,00 \%)$ não se obteve o isolamento de Candida spp nem da mucosa, nem da prótese, conforme também observado por com Senna ${ }^{21}$, que relatou a não isolamento em $6,56 \%$ dos casos.

Muitos desinfetantes químicos são sugeridos para a higienização e desinfecção das próteses. O hipoclorito de sódio é de custo 
acessível, tem um grande espectro de ação e requer um curto período de contato sendo que para além dos microrganismos do biofilme, têm capacidade de desinfecção dos microrganismos que ficam aderidos nos poros da superfície da prótese $^{25}$. No entanto, possui algumas desvantagens, destacando-se a atividade corrosiva da superfície dos metais, ação irritante das células e destruição de tecidos. Os desinfetantes que contêm gluteraldeído têm como vantagens o fato de não serem inativados no contato com substâncias orgânicas, não serem corrosivos, não degradarem materiais borrachóides, estando a sua eficácia associada com o período de exposição ${ }^{25}$. No presente estudo observou-se menor prevalência de colonização em pacientes que fazem escovação associada ao molho no hipoclorito.

A associação de higienização inadequada de prótese e estomatite foi mostrada por Kim et al. $^{26}$, que constataram significativa melhora clínica após a substituição das próteses. Entre 39 indivíduos usando próteses totais e com estomatite protética, mais de $80 \%$ não realizavam higienização eficiente das próteses, e 100\% apresentaram colonização de suas próteses por $C$. albicans. A estomatite protética foi eliminada em quase dois terços dos indivíduos após a troca das próteses e execução de práticas corretas de higiene ${ }^{26}$

A medida preventiva mais importante, talvez seja ao controle do ambiente oral, particularmente no que se refere às ações que tendem a diminuir a necessidade de próteses totais $^{20}$. A higienização diária e desinfecção das próteses dentárias removíveis é necessária para promover a saúde e conservação dos tecidos orais. O presente estudo também demonstrou maior colonização das próteses do que das mucosas orais, o que pode indicar que a manutenção da saúde da mucosa está relacionada com o grau de limpeza das próteses em contato com tecidos orais ${ }^{20}$.

\section{CONCLUSÃO}

Os resultados do presente estudo permitem concluir que a prevalência de Candida spp foi de $70,6 \%$ entre os pacientes usuários de próteses removíveis; que a $C$. albicans apresenta maior prevalência em próteses e menor prevalência em mucosas orais; e que as espécies mais prevalentes que colonizaram as próteses e mucosas, em ordem decrescente, foram: $C$. albicans, $C$. glabrata, $C$. parapsilosis, C. tropicalis, e Candida spp.

\section{CONFLITO DE INTERESSE}

Os autores declaram não haver qualquer potencial conflito de interesse que possa interferir na imparcialidade deste trabalho científico.

\section{REFERÊNCIAS}

1. Moreira D, Spolidório DM, Rodrigues JA, Boriollo MF, Pereira CV et al. Candida spp. biotypes in the oral cavity of school children from different socioeconomic categories in Piracicaba-SP, Brazil. Pesqui Odontol Bras. 2001;15(3):187-95. DOI: http://dx.doi.org/10.1590/S151774912001000300003

2. Willis AM, Coulter WA, Fulton CR, Hayes JR, Bell $\mathrm{PM}$ et al. Oral candidal carriage and infection in insulin-treated diabetic patients. Diabet Med. 1999;16(8):675-9.

DOI: http://dx.doi.org/10.1046/i.1464-5491.1999.00134.x

3. Mesquita RA, Aguiar MCF, Tarquino SBC. Candidiase oral com a infecção HIV. Rev Gramg. 1998;4:27-31.

4. Laskaris G, Hadjivassiliou M, Stratigos J. Oral signs and symptoms in $160 \mathrm{HIV}$ - infected patients. J Oral Pathol Med. 1992;21:120-3.

DOI: http://dx.doi.org/10.1111/j.16000714.1992.tb00994.x

5. Arendorf TM, Walker DM. The prevalence and intra-oral distribution of Candida albicans in man. Arch Oral Biol. 1980;(25):1-10. DOI: http://dx.doi.org/10.1016/0003-9969(80)90147-8

6. Souza LB, Pinto LP, Medeiros AMC, Araújo Jr RF, Mesquita OJX. Manifestações orais em pacientes com AIDS em uma população brasileira. Pesq Odont Bras. 2000;4:79-85. DOI: http://dx.doi.org/10.1590/S151774912000000100014

7. Wingard JR. Importance of Candida species other than C. albicans as pathogens in oncology patients. Clin Infect Dis. 1995;20:115-25. DOI: http://dx.doi.org/10.1093/clinids/20.1.115

8. Kirkpatrick WR, Lopez-Ribot JL, McAtee RK, Patterson TF. Growth competition between Candida dubliniensis and Candida albicans under broth and 
biofilm growing conditions. J Clin Microbiol. 2000;38(2):902-4.

9. El-Azizi MA, Starks SE, Khardori N. Interactions of Candida albicans with other Candida spp. and bacteria in the biofilms. J Appl Microbiol. 2004;96:1067-73.

DOI: http://dx.doi.org/10.1111/i.1365-2672.2004.02213.x

10. Branting C, Sund ML, Linder LE. The influence of Streptococcus mutans on adhesion of Candida albicans to acrylic surfaces in vitro. Arch Oral Biol. 1989;34:347-53. DOI: http://dx.doi.org/10.1016/0003-9969(89)90108-8

11. Li L, Redding S, Dongari-Bagtzoglou A. Candida glabrata: an emerging oral opportunistic pathogen. J Dent Res. 2007;86:204-15.DOI: http://dx.doi.org/10.1177/154405910708600304

12. Penha SS, Birman EG, Silveira FRX, Paula CR. Frequency and enzymatic activity (proteinase and phospholipase) of Candida albicans from edentulous patients, with and without denture stomatitis. Pesqui Odontol Bras. 2000;14:119-22, https://doi.org/10.1590/S1517-74912000000200005

13. Theilade E, Budtz-Jorgensen E. Predominant cultivable microflora of plaque on removable dentures in patients with denture-induced stomatitis. Oral Microbiol Immunol. 1988;3:8-13. DOI: http://dx.doi.org/10.1111/j.1399302X.1988.tb00597.x

14. Kokjonh K., Bradley, M., Griffitha, B., Ghannoum, M. Evaluation of in vitro activity of ciclopirox olamina, butenafine $\mathrm{HCL}$ and econazole against dermathophytes and bacteria. International Journal of Dermatology, 2003. 42, p.11-7. DOI: http://dx.doi.org/10.1046/i.1365-4362.42.s1.4.x

15. Lacaz, CS, Porto E, Martins JEC, Heins-Vaccari EM, Melo NT. Tratado de Micologia médica. 9.ed. São Paulo: Sarvier; 2002.

16. Urizar, JMA. Candidiasis orales. Rev Iber Micol. 2002;19:17-21.

17. Lemos MMC, Miranda JL.; Souza MSGS. Estudo clínico, microbiológico e histopatológico da estomatite por dentadura. Rev Bras Patol Oral. 2003;2(1):3-10.

18. Pereira-Cenci T, Fernandes FS, Skupien JA, Mesko $\mathrm{ME}$, Straioto FG, Del Bel Cury AA. Can new dentures decrease Candida Levels? Int J Prosthod. 2013;26(5):470-7. DOI:
19. Valentini F, Luz MS, Boscato N, Pereira-Cenci T. Biofilm formation on denture liners in a randomized controlled in situ trial. J Dentist. 2013;41(5):420-7. DOI: http://dx.doi.org/10.1016/j.jdent.2013.02.012

20. Bianchi CMPC, Bianchi EA, Tadano T, Paula CR, Santos HDH, Leite Jr DP, Hahn RC. Factors related to oral candidiasis in elderly users and non-users of removable dental prostheses. Rev Inst Med Trop. 2016;58(17):1-5. DOI: http://dx.doi.org/10.1590/S1678-9946201658017.

21. Senna AM. Terapia Fotodinâmica Antimicrobiana no Tratamento da Estomatite Protética. [Tese]. Universidade de São Paulo, Instituto de Pesquisas Energéticas e Nucleares, Programa de Pós-graduação em Tecnologia Nuclear - Materiais, 2012.

22. Teixeira ML, Mezzari A. Prevalência de Candida albicans e Candida não-albicans em próteses dentárias. NewsLab. 2005;70:116-22.

23. Leite DP, Piva MR, Martins-Filho PRS. Identificação das espécies de Candida em portadores de estomatite protética e avaliação da susceptibilidade ao miconazol e à terapia fotodinâmica. Rev Odontol UNESP. 2015;44(1):12-7. Doi: http://dx.doi.org/10.1590/1807-2577.1027

24. Gasparetto A, Negri MFN, Paula CR, Svidzinski TIE. Produção de biofilmes por leveduras isoladas da cavidade bucal de usuários de prótese dentária. Acta Sci Health Sci. 2005;27(1):37-40. DOI: $\underline{\text { http://dx.doi.org/10.4025/actascihealthsci.v27i }}$ $\underline{1.1437}$

25. Gusmão JMR. Leveduras do gênero Candida na saliva de usuários de prótese parcial removível a grampo. [Dissertação]. Taubaté: Universidade de Taubaté, Pós-graduação em Odontologia, 2007.

26. Kim E, Driscoll CF, Minah GE. The Effect of a denture adhesive on the colonization of Candida Species in vivo. J Prosthodont 2003;12:187-91. DOI: http://dx.doi.org/10.1016/S1059$\underline{941 \times(03) 00050-0}$

Recebido para publicação em 16/08/2017

Revisado em 15/09/2017

Aceito em 17/09/2017

http://dx.doi.org/10.11607/ijp.3047 